\title{
Patellofemoral Outcome after Medial Unicompartmental Knee Replacement: A Retrospective Study of 25 Cases
}

\author{
Nader Naouar, Hamdi Kaziz, Thabet Mouelhi, Mohamed Amine Triki, Walid Osmane, \\ Mohamed Laziz Ben Ayeche
}

Orthopedics Department, Sahloul University Hospital, Sousse, Tunisia

Email: hamdi.kaziz@gmail.com

How to cite this paper: Naouar, N., Kaziz, H., Mouelhi, T., Triki, M.A., Osmane, W. and Ayeche, M.L.B. (2017) Patellofemoral Outcome after Medial Unicompartmental Knee Replacement: A Retrospective Study of 25 Cases. Open Journal of Orthopedics, 7, 320-328.

https://doi.org/10.4236/ojo.2017.710032

Received: July 8, 2017

Accepted: October 13, 2017

Published: October 16, 2017

Copyright () 2017 by authors and Scientific Research Publishing Inc. This work is licensed under the Creative Commons Attribution International License (CC BY 4.0).

http://creativecommons.org/licenses/by/4.0/

\begin{abstract}
Introduction: Osteoarthritis and impingement, in patellofemoral compartment after partial arthroplasty, have been rarely reported. The purpose of our study was to evaluate outcome at long-term follow-up. Methods: We have reviewed retrospectively twenty-five unicompartmental arthroplasties. The medial parapatellar approach was used in all cases. Patients have been evaluated with regard to patellar impingement and osteoarthritic changes revealed by radiographs. The mean follow-up was 10.4 years. The clinical scoring system of the Knee Society and specific questions regarding patellofemoral symptoms were used for functional outcome. Patellofemoral complications such as osteoarthritis, impingement and the position of the femoral component were diagnosed on X-ray views of the knee. Results: At the final follow-up, fifteen knees revealed lateral patellofemoral osteoarthritis and four knees had patellofemoral impingement. Excepted of two cases, all knees with patella impingement did not revealed degenerative modifications. Anterior pain of the knee was noted more frequently in knees with patellar complications. Patellar impingement was considered the first cause of knee pain, more than knees with degenerative modifications. Only one revision surgery was indicated in front of patellofemoral conflict. The occurrence of patellar impingement depends on the surgical procedure. The anterior position of the femoral component seems to increase the frequency of such complication. Conclusion: Several degenerative modifications may occur in the patello-femoral compartment following partial knee arthroplasty. Patellar impingement and total knee arthritis are the most reported modifications. Functional outcome may be affected progressively when these changes become obvious. Patellar impingement is the major cause of knee pain and needs revision.
\end{abstract}




\section{Keywords}

Patellofemoral, Unicompartimental, Arthroplasty, Outcome

\section{Introduction}

In spite of partial knee replacement, degenerative modifications continue to occur in all joint's compartments. Reviewing the literature, some especial complications have been reported such as major modifications in patellofemoral compartment. In fact, patellofemoral compartment may be affected after medial partial knee arthroplasty. Some especial complications may occur such as patellar impingement and osteoarthritis resulting from the evolution of the degenerative disease of the knee. Reviewing literature, these complications have been rarely reported after medial replacement of the knee. Furthermore, the long-term outcome was not well established. The objective of our study was to provide the frequency of patellofemoral osteoarthritis and impingement after medial replacement of the knee and their influence on the long-term functional outcome, particularly with regard to pain and other symptoms specific of the patellofemoral compartment.

\section{Materials and Methods}

We have reviewed retrospectively twenty-five medial knee arthroplasties. All knees were operated between January 1993 and December 2003. Inclusion criteria were: tibiofemoral arthritis less or equal to grade two of Ahlback classification [1], degenerative etiology of the knee arthritis and presence of patient at final follow-up. Exclusion criteria were presence of anatomical abnormalities of the distal extremity of the femur, posttraumatic knee arthritis, patients suffering from rheumatoid knee arthritis and vascular problems of lower limb. Three experienced surgeons have operated all patients according the same procedure. Parapatellar approach was used in all cases. All patients were followed with same instructions on postoperatively. All knees were managed with the same medial unicompartmental prosthesis. All knees were evaluated clinically with use of the Knee Society score [2]. Patellofemoral compartment was evaluated and graded according a specific scale [3] divided into 3 grades: grade 0: there was no pain with stair-climbing or rising from a chair; grade 1: mild pain; and grade 2: moderate or severe pain. At final follow-up, X-ray views were used to evaluate modifications involving the patellofemoral joint. Skyline radiographs with a respective flexion of the knee at $30^{\circ}$ and $90^{\circ}$ respectively, were used according to the method described by Merchant [4]. Preoperative radiographic evaluation of the called sunrise was based on Laurin's method [5]. The preoperative congruity of the patellar joint was classified as type I (normal), type II (shifted), type III (tilted), or type IV (shifted and tilted). Patellofemoral osteoarthritis was analyzed on the radiographs. Some typical modifications have been searched such as pa- 
tellar subchondral sclerosis, subchondral cyst formation, or narrowing of the patellofemoral joint. Patellar impingement commonly called notch in radiographic views was defined as a contact between the femoral metal component and the native patellar cartilage. Measurement of the depth and width of the notch on the patellar facet was assessed when there was an erosive loose of patellar cartilage (Figure 1). In all cases, Insall-Salvati index [6] was used to classify patella position. To analyze impingement, the position of the femoral component was explored in both sagittal and coronal X-ray views. The inclination of the femoral component was determined with respect to the femoral axis. The distance from the anterior part of the component to the line of the slope of the intercondylar roof was measured (Figure 2). This distance was positive when the anterior edge of the femoral component was anterior to the line of the slope of the intercondylar roof and negative when the anterior edge was posterior to the line. The same measurements were made on the preoperative radiographs, with the junction between the trochlea and the condyle used instead of the anterior edge of the femoral implant. The preoperative and postoperative distances were compared to assess the anteroposterior position of the femoral component with regard to the trochleocondylar junction. To obtain the exact measurement (in millimeters) of the position of the anterior edge of the femoral component in relation to the trochleocondylar junction, the size of the femoral component on radiographs was used to determine the magnification. The level of significance was set at 0.05. SPSS for Windows (version 19.0, SPSS, Chicago, IL), was used for the statistical analysis. The chi-square test was used to identify trends within groups with categorical variables.

\section{Results}

The mean age was 67.4 years ( $\min 54$ years, max 72 years). The sex ratio was 0 , 78 (male: 11, female: 14). At final follow-up, five knees had been revised. The

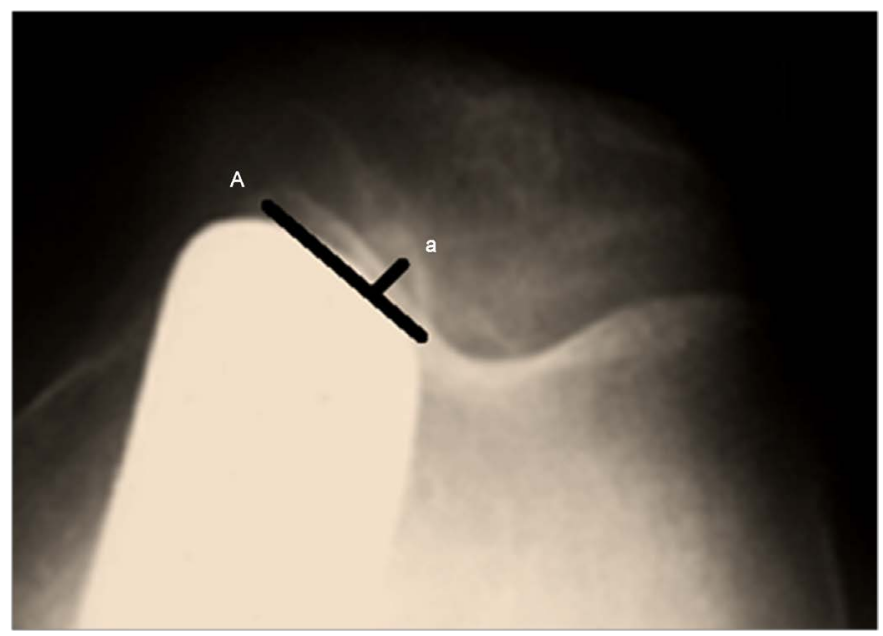

Figure 1. Patellofemoral view of the knee showing the procedure using for the measurement of both the depth and width of the notch on the patella. 


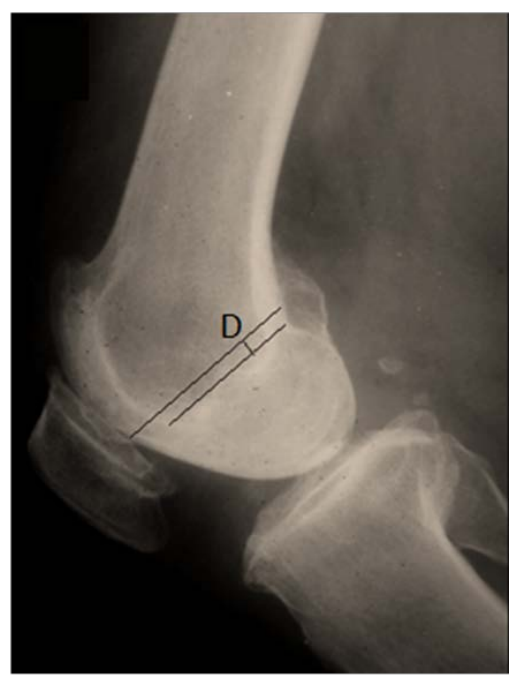

(a)

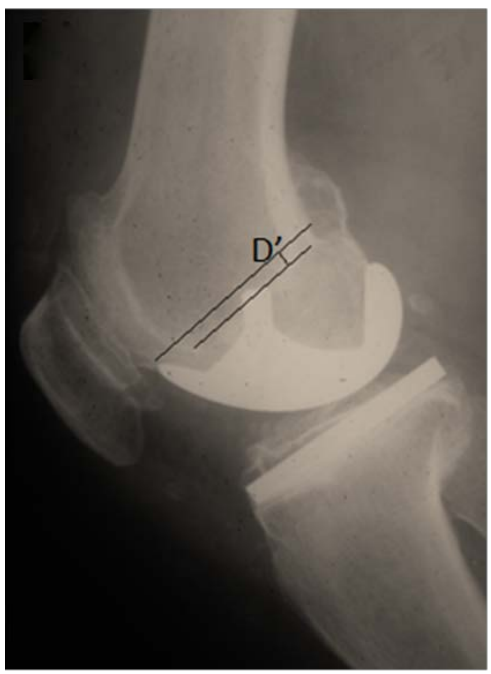

(b)

Figure 2. Diagram depicting the method used to measure the position of the anterior part of the femoral component and the position of the trochleocondylar junction on radiographs. $\mathrm{D}=$ distance, in millimeters, from the anterior part of the component to the line of the slope of the intercondylar roof.

average time to revision was 8.7 years (range, one to fourteen years).The mean follow-up was 10.4 years. The mean preoperative and postoperative knee scores were respectively 68.8 points and 88.7 points. The preoperative and postoperative functional scores were respectively 67.2 points and 82.3 points (Table 1 ). The reasons for revision included infection (one knee), loosening of the polyethylene bearing component (one knee), degeneration in the opposite compartment (two knees), and patellar impingement (one knee) (Table 2). Impingement of the femoral metal component on the patella was observed on the skyline radiograph obtained with flexed knee at $90^{\circ}$ in 4 cases. Fifteen knees have revealed radiographic modifications in patellofemoral joint such as narrowing of the joint space, erosive cartilage and sclerosis. All our cases presenting an impingement have been diagnosed to present osteoarthritis of the patellofemoral compartment which was well objective through the axial radiograph made with the knee in $30^{\circ}$ of flexion. Ten knees demonstrated osteoarthritic changes in the lateral tibiofemoral compartment. In fact, at the time of the most recent follow-up, all knees were divided in four groups depending on the status of the patellofemoral joint: Group I had a normal patellofemoral joint, Group II had osteoarthritic changes on the opposite (medial or lateral) side of the patellofemoral joint with respect to the implant, and Group III had patellar impingement on the side of the patellofemoral joint and Group IV had both osteoarthritis and impingement (Figure 3). Patellofemoral symptoms were analyzed in all knees. Four knees had patellar impingement, and fifteen knees had degenerative changes. The mean stair-climbing score after medial arthroplasty was respectively 48 (range, 40 to 50 points) in Group I; 43 points (range, 40 to 50 points) in Group II; 34 points (range, 30 to 40 points) in Group III and 30 points in Group IV. The difference 
Table 1. Socio-demographic and clinical characteristics of the population.

\begin{tabular}{cc}
\hline Characteristics & Mean \\
Age & 67.4 years \\
Sex-ratio & 0.78 \\
& $($ min: 54 , max: 72$)$ \\
BMI & 22.6 \\
Knee score & 68.8 points \\
Preoperative & 88.7 points \\
Postoperative & \\
Functional score & 67.2 points \\
Preoperative & 82.3 points \\
Postoperative & 10.4 years \\
Follow-up & 8.7 years \\
Delay of revision & $($ min: 1 , max: 14$)$ \\
\hline
\end{tabular}

Table 2. Complications and their treatment.

\begin{tabular}{|c|c|c|}
\hline Complications & Number & Treatment \\
\hline Infection & 1 & Surgical debridement \\
\hline $\begin{array}{l}\text { Degenerative modification of lateral } \\
\text { compartment }\end{array}$ & 2 & Total knee replacement \\
\hline Loosening of PE bearing component & 1 & Total Knee replacement \\
\hline Patellar impingement & 4 & Only 1 total knee replacement \\
\hline
\end{tabular}

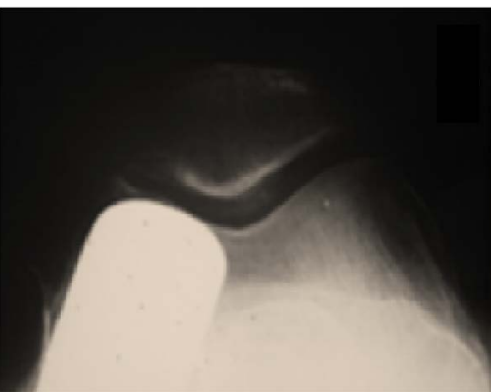

(a)

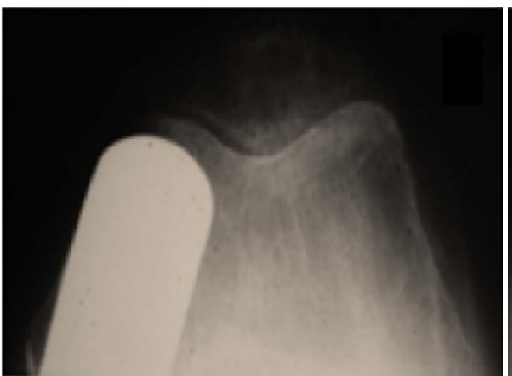

(c)

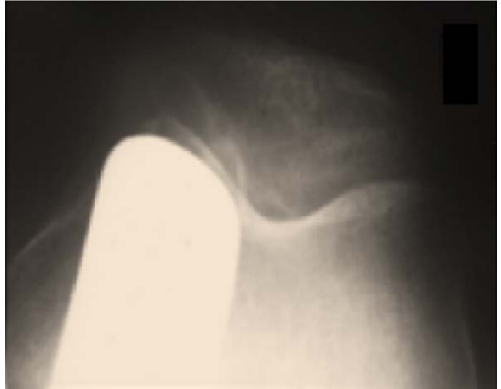

(b)

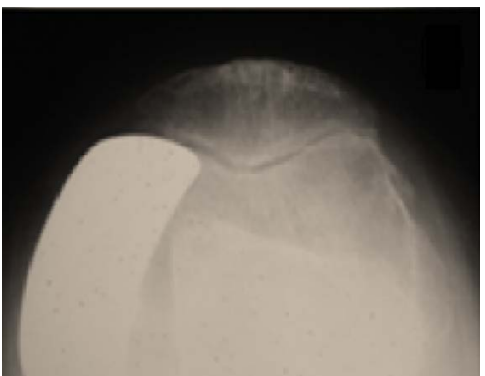

(d)

Figure 3. The status of the patellofemoral joint: (a) Normal patellofemoral joint; (b) Osteoarthritic changes on the medial side of the patellofemoral joint with respect to the implant; (c) Patellar impingement on the side of the patellofemoral joint and (d) Both osteoarthritis and impingement. 
was significant between Groups I and IV $(\mathrm{p}=0.03)$ and between Groups II and III $(p=0.02)$. This finding demonstrates that knees with a normal patellofemoral joint did better than knees with osteoarthritic changes and that both of those groups did better than knees with patellar impingement. Patellar impingement was also observed in all knees that had severe (grade 2) pain during stair climbing or rising from a chair ( $\mathrm{p}=0.02$, chi-square test). Radiographic Findings had never revealed joint space narrowing on the preoperative axial skyline radiograph. The grade of joint congruity on the preoperative axial skyline radiograph was a significant predictor of the joint space narrowing seen at the time of the most recent follow-up. Knees that had normal congruity of the patellofemoral joint on the preoperative radiograph were less likely to have degenerative changes at the time of the most recent follow-up. There was a significant difference between normal and osteoarthritic knees with regard to the preoperative congruity of the patellofemoral joint ( $\mathrm{p}=0.001$, chi-square test). The severity of the erosive changes was a significant predictor of the patellofemoral symptoms in the knees with patellar impingement. Severe changes were defined as those that were $>5 \mathrm{~mm}$ in depth or $>10 \mathrm{~mm}$ in width. The five knees with severe erosive changes had grade 2 symptoms, and the four knees with slight erosive changes had grade 1 symptoms $(\mathrm{p}=0.001)$. Four knees in which the femoral component had been placed in at least $10^{\circ}$ of extension had impingement of the patella. This finding was related to insufficient posterior bone resection resulting in placement of the femoral component in extension, which produced slight anterior projection of the femoral component on the axial skyline radiograph. Impingement of the patella was not observed in any knees in which the femoral component was not anterior to the preoperative trochleocondylar junction. In comparison, impingement was observed in four knees in which the femoral component was at least $5 \mathrm{~mm}$ anterior to the junction. Impingement of the patella was associated with anterior placement of the femoral component ( $\mathrm{p}=$ 0.001). Small femoral implant was found to be cemented in all knees with impingement. Only one knee had needed a revision surgery and total knee arthroplasty was indicated in front of severe pain. Two knees had a revision because of patellofemoral symptoms associated with generalized arthritis of both patellofemoral and tibiofemoral compartments. One patient had been operated for polyethylene loosening and a total knee replacement had been indicated (Figure 4 ). The low number of revisions performed for patellar impingement was probably related to the advanced age of the patients (average, eighty-four years at the time of the last follow-up).

\section{Discussion}

The purpose of our study was to report major complications occurring at long term follow-up of partial medial knee arthroplasty. The most observed complication is osteoarthritis in the patellofemoral joint [7] [8]. Impingement was observed in four knees in which only one knee was revised in front of major pain 


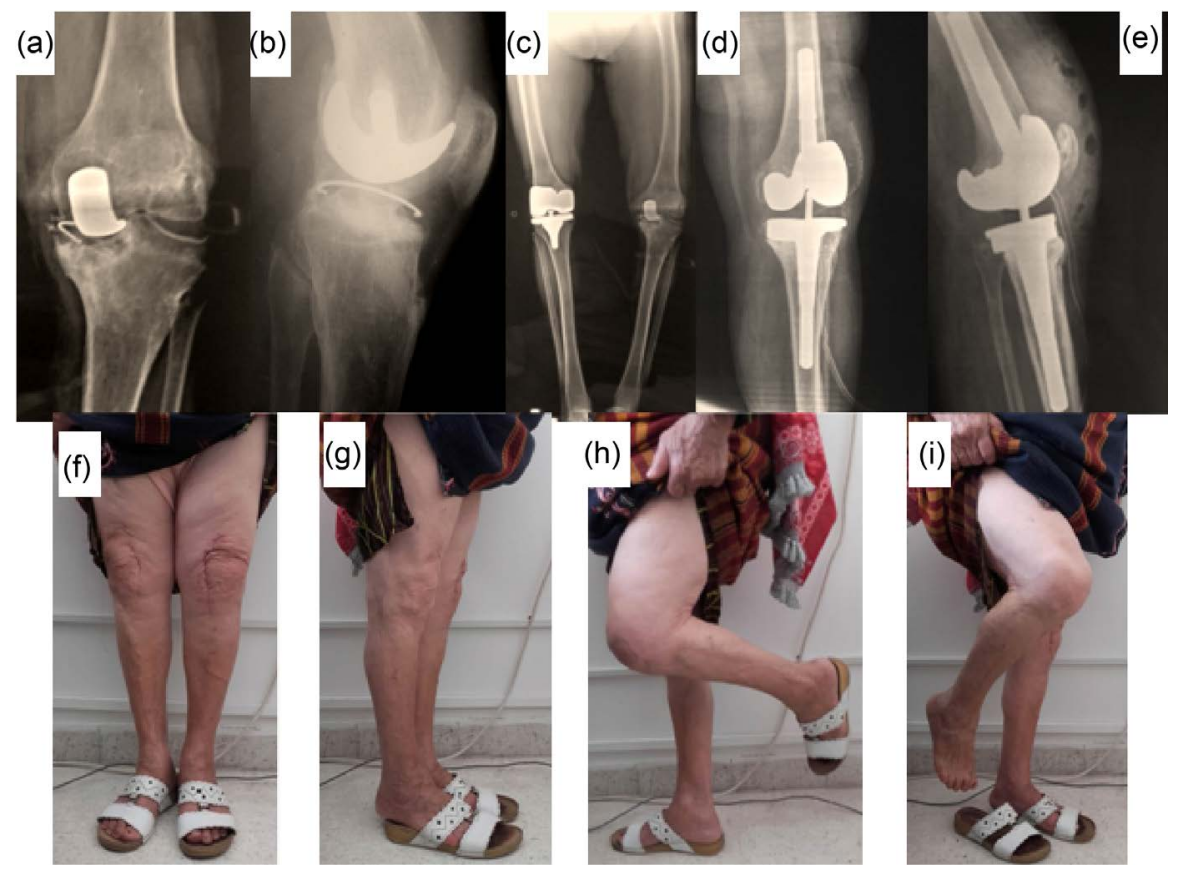

Figure 4. Revision of medial unicompartmental knee arthroplasty due to polyethylene loosening and femoropatellar impingement: (a) (b) Antero-posterior and lateral views of the left knee revealing the polyethylene loosening and the femoropatellar impingement explained by the conflict of anterior part of femoral component and the patella; (c) Weight-bearing bilateral long-leg standing radiograph showed a preoperative left HKA at $171^{\circ}$ and right HKA at right $178^{\circ}$; (d) (e) Postoperative antero-posterior and lateral X-rays views of left knee, total knee arthroplasty was indicated after removal of the femoral component and tibial component; (f) (g) (h) (i) Functional results at final outcome: the patient didn't complain from pain, no axial abnormalities were diagnosed with $90^{\circ}$ flexion of the left knee.

and incapacity. A comparison between knees without patellar impingement and knees with patellar impingement showed significant differences with regard to the stair-climbing component of the Knee Society score and with regard to the presence of pain during stair-climbing or rising from a chair. Patellofemoral impingement was associated with anterior placement of the femoral component [8]. The transitional zones between the trochlea and the medial and lateral condyles (that is, the zones of medial and lateral meniscal impression on the femur) were not situated at the same levels on the preoperative radiographs. Preoperatively, the trochleocondylar junction was an average of $3 \mathrm{~mm}$ anterior to the line of the slope of the intercondylar roof on the medial side whereas it was an average of $4 \mathrm{~mm}$ posterior to this line on the lateral side (Figure 2). This can occur when too much bone is resected from the posterior part of the condyle, bringing the anterior portion of the component forward, or there is a poor assessment of the location of the trochleocondylar junction, which is sometimes difficult to identify during the operation because of the presence of large osteophytes [8] [9]. Posterior bone resection with the implant in too much extension can also result in an abnormal prominence of the implant at the time of cementing. Several studies prove that impingement is more seen in lateral knee arthroplasty [8] 
[9] [10] [11]. Impingement of the patella is a specific complication of unicompartmental arthroplasty that may lead to patellofemoral symptoms and may affect the ability to resurface the patella during a subsequent revision to a total knee arthroplasty [12]. Degenerative changes also affect the long-term outcome of unicompartmental arthroplasty, particularly when there is abnormal joint congruity on preoperative radiographs [13] [14] [15]. In our series, these changes may have been related to the absence of lateral retinacular release or patellar realignment. It is not clear why impingement and osteoarthritic changes appeared to have been mutually exclusive [16] [17]. Since impingement is associated with anterior placement of the femoral component, this complication may protect the opposite portion of the patella by reducing the contact pressures on that side [18] [19] [20].

\section{Acknowledgements}

The authors did not receive grants or outside funding in support of their research or preparation of this manuscript. They did not receive payments or other benefits or a commitment or agreement to provide such benefits from a commercial entity. No commercial entity paid or directed, or agreed to pay or direct, any benefits to any research fund, foundation, educational institution, or other charitable or nonprofit organization with which the authors are affiliated or associated.

\section{References}

[1] Ahlback, S. (1968) Osteoarthrosis of the Knee. A Radiographic Investigation. Acta Radiologica: Diagnosis, 277, 7-72.

[2] Insall, J.N., Dorr, L.D. and Scott, R.D. (1989) Rationale of the Knee Society Clinical Rating System. Clinical Orthopaedics, 238, 159-166. https://doi.org/10.1097/00003086-198911000-00004

[3] Stern, S.H. and Insall, J.N. (1990) Total Knee Arthroplasty in Obese Patients. The Journal of Bone and Joint Surgery, American Volume, 72, 1400-1404. https://doi.org/10.2106/00004623-199072090-00020

[4] Merchant, A.C., Mercer, R.S., Jacobsen, R.H. and Cool, C.R. (1974) Roentgenographic Analysis of the Patellofemoral Congruence. The Journal of Bone and Joint Surgery, American Volume, 56, 1391-1396. https://doi.org/10.2106/00004623-197456070-00007

[5] Laurin, C.A., Dussault, R. and Levesque, H.P. (1979) The Tangential X-Ray Investigation of the Patellofemoral Joint: X-Ray Technique, Diagnostic Criteria and Their Interpretation. Clinical Orthopaedics, 144, 16-26.

[6] Insall, J. and Salvati, E. (1971) Patella Position in the Normal Knee Joint. Radiology, 101, 101-104. https://doi.org/10.1148/101.1.101

[7] Bernasek, T.L., Rand, J.A. and Bryan, R.S. (1988) Unicompartmental Porous Coated Anatomic Total Knee Arthroplasty. Clinical Orthopaedics, 236, 52-59. https://doi.org/10.1097/00003086-198811000-00007

[8] Callahan, C.M., Drake, B.G,. Heck, D.A. and Dittus, R.S. (1994) Patient Outcomes Following Unicompartmental Total Knee Replacement. A Meta-Analysis. JAMA, 271, 1349-1357. https://doi.org/10.1001/jama.1994.03510410061034 
[9] Hernigou, P. and Goutallier, D. (1988) GUEPAR Unicompartmental Lotus Prosthesis for Single-Compartment Femorotibial Arthrosis. A Five- to Nine-Year Follow-Up Study. Clinical Orthopaedics, 230, 186-195.

[10] Hernigou, P. and Deschamps, G. (2002) Patellar Impingement Following Unicompartmenta Arthroplasty. The Journal of Bone and Joint Surgery, American Volume, 84-A, 1132-1137. https://doi.org/10.2106/00004623-200207000-00006

[11] Volpi, P., Marinoni, L., Bait, C., Galli, M. and Denti, M. (2007) Lateral Unicompartimental Knee Arthroplasty: Indications, Technique and Short-Medium Term Results. Knee Surgery, Sports Traumatology, Arthroscopy, 15, 1028-1034. https://doi.org/10.1007/s00167-007-0342-8

[12] McAuley, J.P., Engh, G.A. and Ammeen, D.J. (2001) Revision of Failed Unicompartimental Knee Arthroplasty. Clinical Orthopaedics and Related Research, 392, 279-382. https://doi.org/10.1097/00003086-200111000-00036

[13] Froimson, M.I., Bloomfield, M.R. and Sherman, R.A. (2009) Revision of the Failed Unicompartimental Knee Arthroplasty. Semin Arthroplasty, 20, 23-28. https://doi.org/10.1053/j.sart.2008.11.007

[14] Mozella Ade, P., Borges Gonçalves, F., Osterno Vasconcelos, J. and de Araújo Barros Cobra, H.A. (2014) Revision of Unicompartmental Knee Arthroplasty: Implants Used and Causes of Failure. Revista Brasileira de Ortopedia, 49, 154-159.

[15] Ollivier, M., Parratte, S. and Argenson, J.N. (2013) Results and Outcomes of Unicompartmental Knee Arthroplasty. Orthopedic Clinics of North America, 44, 287-300.

[16] Berger, R.A., Meneghini, R.M., Sheinkop, M.B., et al. (2004) The Progression of Patellofemoral Arthrosis after Medial Unicompartmental Replacement: Results at 11 to 15 Years. Clinical Orthopaedics and Related Research, 428, 92-99. https://doi.org/10.1097/01.blo.0000147700.89433.a5

[17] Beard, D.J., Pandit, H., Ostlere, S., et al. (2007) Pre-Operative Clinical and Radiological Assessment of the Patellofemoral Joint in Unicompartmental Knee Replacement and Its Influence on Outcome. The Journal of Bone and Joint Surgery, 89, 1602-1607. https://doi.org/10.1302/0301-620X.89B12.19260

[18] Munk, S., Odgaard, A., Madsen, F., et al. (2011) Preoperative Lateral Subluxation of the Patella Is a Predictor of Poor Early Outcome of Oxford Phase-III Medial Unicompartmental Knee Arthroplasty. Acta Orthopaedica, 82, 582-588. https://doi.org/10.3109/17453674.2011.618915

[19] Waldstein, W., Jawetz, S.T., Farshad-Amacker, N.A., et al. (2014) Assessment of the Lateral Patellar Facet in Varus Arthritis of the Knee. Knee, 21, 920-925.

[20] Song, E.K., Park, J.K., Park, C.H., et al. (2016) No Difference in Anterior Knee Pain after Medial Unicompartmental Knee Arthroplasty in Patients with or without Patellofemoral Osteoarthritis. Knee Surgery, Sports Traumatology, Arthroscopy, 24, 208-213. https://doi.org/10.1007/s00167-014-3367-9 